Title: Association of socioeconomic status and consumption of cigarettes, illicit drugs, and alcohol

Running title: Socioeconomic status and use of illicit drugs

Maryam Marzban', Maryam Hadji², Mahin Gholipour ${ }^{3}$, Hamideh Rashidian ${ }^{4}$, Abbas

Rezaianzadeh $^{5 *}$, Jafar Hasanzadeh ${ }^{5}$, Ali Akbar Haghdoost 6, Afarin Rahimi-

Movaghar $^{7}$, Reza Ghiasvand ${ }^{8}$, Abdolvahab Moradi', Hossein Khavari-Daneshvar ${ }^{2}$, Elisabete Weiderpass $^{10}$, Farin Kamangar ${ }^{11}$, Kazem Zendehdel ${ }^{2}$

$1 \mathrm{PhD}$ candidate, Students Research Committee, Shiraz University of Medical Sciences, Shiraz, Iran.

${ }^{2}$ MSC, Cancer Research Center, Cancer Institute of Iran, Tehran University of Medical Sciences, Tehran, Iran.

${ }^{3} \mathrm{MD}$, Golestan Research Center for Gastroenterology and Hepatology, Golestan University of Medical Sciences, Gorgan, Iran.

${ }^{4} \mathrm{PhD}$ candidate, Department of Biostatistics and Epidemiology, Kerman University of Medical Sciences, Kerman, Iran.

${ }^{5}$ MD-PhD, Department of Epidemiology, School of Public Health, Shiraz University of Medical Sciences, Shiraz, Iran.

${ }^{6}$ MD-PhD, Regional Knowledge HUB for HIV/AIDS Surveillance, Research Center for Modelling in Health, Institute for Future Studies in Health, Kerman University of Medical Sciences, Kerman, Iran.

${ }^{7} \mathrm{MD}-\mathrm{PhD}$, Iranian National Center for Addiction Studies (INCAS), Tehran University of Medical Sciences, Tehran, Iran.

${ }^{8}$ MSC-PhD, Oslo Center for Biostatistics and Epidemiology, Institute of Basic Medical Sciences, University of Oslo, Oslo, Norway.

${ }^{9} \mathrm{MD}$, Department of Microbiology, Golestan University of Medical Sciences, Gorgan, Iran.

${ }^{10}$ Department of Medical Epidemiology and Biostatistics, Karolinska Institute, Stockholm,

Sweden; Department of Research, Cancer Registry of Norway, Institute of Population-Based Cancer Research, Oslo, Norway; Genetic Epidemiology Group, Folkhälsan Research Center, Helsinki, Finland; Department of Community Medicine, University of Troms $\varnothing$, The Arctic University of Norway, Troms $\varnothing$, Norway 
${ }^{11}$ MD-PhD, Department of Public Health Analysis, School of Community Health and Policy, Morgan State University, Baltimore.

*Corresponding author

\section{Abbas Rezaianzadeh}

Department of Epidemiology, School of Public Health, Shiraz University of Medical Sciences, Shiraz, Iran.

Email: rezaiana@gmail.com

Tel: 0098-071-37251001

Fax: 0098-071-37260225 


\section{Abstract: \\ Introduction and Aims:}

Socioeconomic Status (SES) is considered as one of the important factors associated with use of various drugs. The present study aimed to investigate the effect of SES on cigarette smoking, alcohol use, drug use, and passive exposure to opium and cigarette smoke.

\section{Design and Methods:}

In this study, which is part of a multicenter case-control study, the research hypothesis was checked among controls who had referred to hospitals. Data were collected through a questionnaire and laboratory tests to determine the actual consumers of opium and other illicit drugs. Then, the data were analyzed using STATA 13.

\section{Result:}

This study was performed on 364 individuals within the age range of 30 to 75 years. More than $55 \%$ of the participants had a history of life-time consumption of cigarettes and hookah as well as alcohol and drugs. The results revealed an inverse relationship between SES and life-time consumption of hookah and alcohol. Furthermore, individuals with higher SES were more likely to deny their drug use.

\section{Discussion and Conclusions:}

The results revealed little robust evidence supporting the assumption that SES level can have an important effect on illicit drug use. On the other hand, the participants' characteristics could have a prominent effect on precise evaluation of the relationship between SES and drug use. Further multicenter studies are needed with samples diversified in terms of age and ethnicity to identify these confounding relationships.

Keywords: Socioeconomic status, Alcohol, Drug use, Cigarette smoking

\section{Introduction:}

Socioeconomic Status (SES) refers to the position held by a person in the structure of the society in terms of social or economic factors (Lynch \& Kaplan, 2000). Nowadays, SES is considered an important factor associated with disabilities, mortality rate, cancer, and other health indicators (Gong, Xu, \& Takeuchi, 2012). In most studies, the association between various indicators and SES has been assessed using objective variables, such as wealth, 
income level, occupation, and education level, each of which entails certain challenges (Banks, Marmot, Oldfield, \& Smith, 2006; Demakakos, Nazroo, Breeze, \& Marmot, 2008). Although the important role of SES in various domains cannot be ignored, there is no agreement on the best indicators that can be used for this purpose (Ahmadpanah et al., 2013).

Substance abuse is one of the most intricate problems in human life (Ahmadpanah et al., 2013). The harm from addictive behaviors varies based on SES (Room, Sankaran, Schmidt, Mäkela, \& Rehm, 2015). A few studies examining the relationship between SES and duration and prevalence of smoking (Nagelhout et al., 2012) in Western countries have indicated that individuals with lower SES have had a longer duration of smoking. However, these findings varied with respect to specific measures of SES and in magnitude of the association between SES and duration and prevalence of smoking (Nagelhout et al., 2012). It should be mentioned that the present time is one of transition; hence, there are various socioeconomic patterns of smoking and heavy smoking around the world. For instance, heavy smoking in high-income countries has a strong inverse relationship with social class, whereas this pattern may often be reversed in low- to middle-income countries.

Moreover, some studies have revealed a complex association between alcohol consumption and SES. Indeed, many studies have found that the burden of alcohol-related mortality and morbidity falls most heavily on individuals with lower SES (Jones, Bates, McCoy, \& Bellis, 2015; Kendler et al., 2014; Probst, Roerecke, Behrendt, \& Rehm, 2014).

Most studies investigating the relationship between alcohol and illegal drug use and SES have been conducted in high-income countries (Room, Sankaran, et al., 2015), including the United States (Humensky, 2010), the United Kingdom, the Netherlands, and Finland (KARRIKER-JAFFE, 2011). The present study aims to investigate the association between SES and illicit drug and alcohol use in Iran as a developing country where the rate of alcohol consumption is low (Amin-Esmaeili et al., 2016), but the prevalence of opium use is highest in the world (Amin-Esmaeili et al., 2016). Another novelty of this study is evaluating the role of passive exposure to opium and cigarette smoke. The association between denied illicit drug use and SES is going to be assessed, as well.

\section{Methods:}

\section{Study population}


We designed a large multicenter case-control study carried out in provinces of Tehran (center of Iran), Kerman (east of Iran), Fars (south of Iran), and Golestan (north of Iran) to evaluate the association between opium consumption and cancer risk (IROPICAN study). In the present study, we used data regarding drug use, alcohol use, and socioeconomic factors from non-cancer patients who were healthy individuals visiting hospitals or patients admitted to hospitals for reasons other than cancer and opium-related diseases. Because the prevalence of opium use is very low among women, only males were recruited in this study. The sample size was calculated based on specificity $(70 \%)$ and sensitivity $(80 \%)$ of the questionnaire to identify consumers and the assumption that diagnostic tests can increase these two indices up to $90 \%$. The participants were selected based on age group and place of residence to obtain a representative sample. Participation rate was high in both healthy $(86 \%)$ and patient controls $(88 \%)$. Non-participants were defined as individuals who refused to participate after the study was explained to them. About half of the non-participation rates were due to unwillingness to provide urine samples in both control groups.

\section{Data collection method}

In this study, questionnaires and laboratory tests were used to collect data on SES and alcohol and drug use. In doing so, interviewers experienced in interviewing individuals with a history of substance use were especially trained in a 2-day workshop to standardize the interviewing process and mitigate inter-rater variability. A detailed questionnaire guideline was used, as well. All participants were invited to interview in a private room. After the interview, urine samples were collected for laboratory analyses.

\section{Socioeconomic status variables}

We used the five-class, self-coded version of National Statistics Socioeconomic Classification (NS-SEC), which included (i) managerial, administrative, and professional occupations, (ii) intermediate occupations, (iii) small employers and own-account workers, (iv) lower supervisory and technical occupations, and (v) semi-routine and routine occupations (Martin \& Deacon, 2000). It should be mentioned that job classification was considered and analyzed as part of the SES index. We decided to generate and validate a new version compatible with the objectives of our study. Thus, we initially collected the expert panel's ideas. Then, Confirmatory Factorial Analysis (CFA), discriminant and convergent validity, and composite reliability were used through the AMOS software to determine these 
indices. CFA is used to determine whether measures of a construct are consistent with a researcher's understanding of the nature of that construct (Harrington, 2009).

The potential SES indicators that were confirmed to be included in Principal Component Analysis (PCA) were marital status, education level, household head's education level, house ownership, house size in square meters $\left(\mathrm{m}^{2}\right)$, ownership of household appliances including personal car, color TV, vacuum cleaner, washing machine, freezer, computer, furniture, and split air conditioner, and various job statuses including job class, self-coded job, and employment status.

\section{Alcohol and drug use}

Data were collected on use of alcohol, tobacco, naswar (sun and heat-dried tobacco leaves, slaked lime, ash from tree bark, and flavoring and coloring agents are mixed together), opium, heroin, heroin crack, methadone, cannabis, and methamphetamine as well as medicines containing codeine and morphine during the last two weeks (to differentiate medical drug use from illegal drug use). Life-time or regular substance users were defined as individuals consuming the substance at least once a week over a six-month period. Each of the aforementioned substances was used in the analysis. In addition, last month or current users were defined as those consuming the substance at least once a week during the month preceding the interview. It should be noted that the study participants were unaware of the research hypothesis. To determine the individuals who denied consumption of the aforementioned drugs, two types of urine drug screens were used; Urine Rapid Drug Screen (URDS) for the initial screening procedure and Thin-Layer Chromatography (TLC) for confirmatory purposes. The TLC test was used for the participants with positive test results on the URDS who denied using opium, heroin, heroin crack, methadone, cannabis, or methamphetamine either regularly or during the past 72 hours in the questionnaires.

\section{Statistical analysis}

PCA was employed as a statistical procedure to convert a set of observations of possibly correlated variables into a set of values of linearly uncorrelated variables for making SES 
index. Numbers and percentages were calculated and presented. Furthermore, logistic regression analysis was used to determine the association between SES and history of drug use and passive exposure to opium and cigarette smoke. Moreover, we used adjusted odds ratio based on place of residence (living in capital city and other cities) and age. It should be mentioned that SES was regarded as a continuous variable (obtained by PCA). All analyses were performed using STATA 13 and AMOS software, version 13. Also, alpha $=0.05$ was considered to be the cutoff value for significance.

\section{Ethical considerations}

This study was approved by the Ethics Committee of Tehran University of Medical Sciences (project code: 93-04-51-27867) and Shiraz University of Medical Sciences (project code: IR.SUMS.REC.1394.S114). All participants signed written inform consents. Additionally, the participants' data were handled confidentially. Urine samples were also kept anonymous for investigators and the results were not linked to the participants' identities in the dataset. Moreover, the participants received a small gift after the interviews.

\section{Results:}

This study was performed on 364 participants. Most of them were married and fewer than one fifth were educated above high school diploma. Additionally, most families had 1 to 4 members and one third of the participants' houses were $100 \mathrm{~m}^{2}$ or smaller in size. The history of life-time cigarette, opium, alcohol, and methadone use was 44\%, 27\%, 15\%, and 4\%, respectively, while that of using other drugs was less than $2 \%$. The results revealed a significant relationship between SES and drug use denial $(\mathrm{OR}=1.30 ; 95 \%$ CI: 1.01; 1.86, pvalue: 0.037). Accordingly, individuals with higher SES were more likely to deny their drug use compared to those with lower SES. Moreover, an inverse relationship was observed between SES and life-time hookah consumption (OR=0.75; 95\% CI: 0.58; 0.96, p-value: $0.024)$ and life-time alcohol use (OR=0.78; 95\% CI: 0.66; 0.91, p-value: 0.002) (Table 1).

\section{Discussion:}


This study was performed to measure SES status, which can be an important factor with an obvious effect on various aspects of life and intricate situations, including addiction. The results revealed a significant relationship between higher SES and alcohol and hookah use. Furthermore, most individuals with high SES tended to conceal their drug use.

So far, numerous studies have been conducted on the association between SES and various health indicators, but there is no agreement on the type of SES indicator (Mohammad et al., 2009). It may be quite difficult to select one or more specific indicators to study the relationship between SES and health outcomes, because some SES markers may have different meanings in different populations or periods of time (Islami et al., 2009).

Iran has one of the highest rates of opioid use in the world and opioid use disorders are the most common type of drug use in Iran (Amin-Esmaeili et al., 2016). The prevalence of opium use is $15-20 \%$ in certain groups of the Iranian population, which is approximately what we found in our study (Nakhaee, Divsalar, Meimandi, \& Dabiri, 2008; Ziaaddini \& Ziaaddini, 2005). However, the prevalence of cigarette smoking was virtually higher in comparison to previous reports (Karimy, Niknami, Heidarnia, Hajizadeh, \& Montazeri, 2013; Nazarzadeh et al., 2013). Nonetheless, the rate of hookah smoking was similar to that reported in other studies (Jones et al., 2015; Probst et al., 2014). Most of the time, these risks are higher for poorer individuals compared to wealthier ones (Room, Sankaran, et al., 2015).

Alcohol can be smuggled into Iran and distributed via black market or is homemade illegally. Nevertheless, a population-based cross-sectional study on Iranian adolescents indicated that $15 \%$ of participants had experienced alcohol use, which is slightly higher compared to the current study findings (Ziaaddini, Ziaaddini, \& Nakhaee, 2013). A previous study showed a diverging relationship between SES and alcohol use depending on the country under investigation (Room, Sujatha Sankaran, Schmidt, \& Rehm, 2015). Iran is a Muslim country where drinking alcohol is presumably stigmatized. On the other hand, due to the legal inhibition in Iran, individuals with high SES have better access to high-quality alcoholic beverages, while those with low SES have access to cheap alcoholic beverages and may use alcohol more. Our results demonstrated that low SES was significantly associated with alcohol and hookah use. Previous studies have also disclosed a significant relationship between SES and alcohol use (Dawson, Grant, Chou, \& Pickering, 1995). A moderate 
relationship was also observed between hookah use and SES in a previous research (Riggs \& Pentz, 2016).

Assessment of SES, illicit drug use, and their relationship is a major limitation in most studies. SES can be evaluated in several different ways and often only at one point in time, which may give a misleading picture of the social environment during various courses of life. SES can also be measured based on both subjective and objective characteristics of individuals and areas (Braveman et al., 2005). Overall, SES is a complicated concept. In this study, SES was regarded as a construct reflecting status and resource distinctions in a given society.

Clear social patterning of a health outcome may occasionally depend on measurement of SES and different predictors that comprise it, such as privation, education, and job class. In the present study, the participants were selected from general hospitals that were focal points and the most important referral centers in the selected provinces and delivered various kinds of services, thus receiving individuals with various SES to these hospitals for treatment. Nevertheless, it is obvious that the study participants' demography would reflect the client population of those particular treatment settings. Even though we had planned to collect participants from the general population and gather samples in the participants' houses, this was not possible because we intended to define denial of consumption accurately. For this purpose, we required valid samples that could only be collected in hospitals' laboratories. Indeed, we tried to recruit participants from various age groups and different parts of the selected provinces. Furthermore, individuals with prominently problematic behaviors may not be inclined to participate in general population-based studies. Therefore, we collected the samples from focal hospitals that serve clients from all classes of the general population. Moreover, we calculated the sample size based on sensitivity and specificity of the questionnaire and laboratory tests and tried to collect samples based on various age groups and places of residency according to a predetermined table. We also collected our data from different parts of the country and various ethnic groups (Kurd, Lor, Persian, etc.), which provided a representative sample of our population.

Generally, underreporting of drug use may occur because respondents fear the possible negative social outcomes or stigmatization (Lam et al., 2015). Hence, drug use should be validated through rapid and complementary tests (TLC) so as to ascertain the number of users 
(Bluth, 2016). The high participation rate in our study could be due to the fact that we employed well-trained interviewers who were strictly supervised. Therefore, few individuals denied their substance use. Based on the results, participants with higher SES were more likely to deny their drug use. To the best of our knowledge, this relationship was not detected in any of the previous studies conducted on the issue. Yet, it is logical that individuals with higher social statuses tend to maintain their prestigious position, thereby covering their faults.

One of the limitations of this study was its small sample size. Although the power of the study was limited, this study was based on the recommendations about prediction models to use 10 events per variable (Greenland, Mansournia, \& Altman, 2016) and the best we could do at the time being. We hope that larger studies will be done in future to confirm these results and provide more precise estimates about the association between SES and illicit drug use. On the other hand, the strong point of this study was that we evaluated the associations between SES and cigarette smoking, illicit drug use, alcohol consumption, and passive exposure to cigarette and opium in Iran, which has the highest rate of opium use and very low rate of alcohol consumption (Room, Sankaran, et al., 2015). Additionally, this was a multicenter study that collected data from different parts of Iran. Moreover, we performed laboratory tests to determine the actual consumers of opium and other illicit drugs, which is prone to underreporting by the general population.

\section{Conclusion:}

In conclusion, the study results revealed little robust evidence supporting the assumption that SES level can have an important effect on illicit drug use. The type of variables and indicators had an important effect on measurement of SES. On the other hand, the characteristics of the study participants could have a prominent effect on precise evaluation of the relationship between SES and drug use. Further multicenter studies are needed with samples diversified in terms of age and ethnicity to identify the confounding relationship between SES and consumption of alcohol and illicit drugs. 


\section{Acknowledgement:}

The present article was extracted from a thesis written by Maryam Marzban, which was funded by Shiraz University of Medical Sciences (grant No. 94-7650) and National Institute for Medical Research Development (NIMAD) (grant No. 940045). Hereby, the authors would like to thank Ms. A. Keivanshekouh at the Research Improvement Center of Shiraz University of Medical Sciences for improving the use of English in the manuscript.

\section{Conflict of Interest:'}

The authors declare that they have no conflict of interests.

\section{References:}

Ahmadpanah, M., Allahverdipour, H., Jalilian, F., Haghighi, M., Afsar, A., \& Gharibnavaz, H. (2013). Effectiveness of Coping Skills Education Program to Reduce Craving Beliefs among Addicts Referred To Addiction Centers in Hamadan: A Randomized Controlled Trial. Iranian Journal of Public Health, 42(10), 1139-1144.

Amin-Esmaeili, M., Rahimi-Movaghar, A., Sharifi, V., Hajebi, A., Radgoodarzi, R., Mojtabai, R., . . . Motevalian, A. (2016). Epidemiology of illicit drug use disorders in Iran: prevalence, correlates, comorbidity and service utilization results from the Iranian Mental Health Survey. Addiction, 111(10), 1836-1847.

Banks, J., Marmot, M., Oldfield, Z., \& Smith, J. P. (2006). Disease and disadvantage in the United States and in England. Jama, 295(17), 2037-2045.

Bluth, M. H. (2016). Toxicology and Drug Testing, An Issue of Clinics in Laboratory Medicine (Vol. 36): Elsevier Health Sciences.

Braveman, P. A., Cubbin, C., Egerter, S., Chideya, S., Marchi, K. S., Metzler, M., \& Posner, S. (2005). Socioeconomic status in health research: one size does not fit all. Jama, 294(22), 2879-2888.

Dawson, D. A., Grant, B. F., Chou, S. P., \& Pickering, R. P. (1995). Subgroup variation in US drinking patterns: results of the 1992 national longitudinal alcohol epidemiologic study. Journal of substance abuse, 7(3), 331-344.

Demakakos, P., Nazroo, J., Breeze, E., \& Marmot, M. (2008). Socioeconomic status and health: the role of subjective social status. Social science \& medicine, 67(2), 330-340. 
Gong, F., Xu, J., \& Takeuchi, D. T. (2012). Beyond conventional socioeconomic status: Examining subjective and objective social status with self-reported health among Asian immigrants. Journal of behavioral medicine, 35(4), 407-419.

Greenland, S., Mansournia, M. A., \& Altman, D. G. (2016). Sparse data bias: a problem hiding in plain sight. bmj, 352, i1981.

Harrington, D. (2009). Confirmatory factor analysis: Oxford University Press.

Humensky, J. L. (2010). Are adolescents with high socioeconomic status more likely to engage in alcohol and illicit drug use in early adulthood? Substance abuse treatment, prevention, and policy, 5(1), 1.

Islami, F., Kamangar, F., Nasrollahzadeh, D., Aghcheli, K., Sotoudeh, M., Abedi-Ardekani, B., . . . Sepehr, A. (2009). Socio-economic status and oesophageal cancer: results from a populationbased case-control study in a high-risk area. International journal of epidemiology, 38(4), 978-988.

Jones, L., Bates, G., McCoy, E., \& Bellis, M. A. (2015). Relationship between alcohol-attributable disease and socioeconomic status, and the role of alcohol consumption in this relationship: a systematic review and meta-analysis. BMC Public Health, 15(1), 1.

Karimy, M., Niknami, S., Heidarnia, A. R., Hajizadeh, I., \& Montazeri, A. (2013). Prevalence and determinants of male adolescents' smoking in Iran: An explanation based on the theory of planned behavior. Iranian Red Crescent Medical Journal, 15(3), 187-193.

KARRIKER-JAFFE, K. J. (2011). Areas of disadvantage: A systematic review of effects of area-level socioeconomic status on substance use outcomes. Drug and alcohol review, 30(1), 84-95.

Kendler, K. S., Gardner, C. O., Hickman, M., Heron, J., Macleod, J., Lewis, G., \& Dick, D. M. (2014). Socioeconomic status and alcohol-related behaviors in mid-to late adolescence in the Avon longitudinal study of parents and children. Journal of studies on alcohol and drugs, 75(4), 541-545.

Lam, L. P., Leung, W. C., Ip, P., Chow, C. B., Chan, M. F., Ng, J. W. Y., . . Chow, K. M. (2015). Validation of the Drug Abuse Screening Test (DAST-10): A study on illicit drug use among Chinese pregnant women. Scientific reports, 5.

Lynch, J., \& Kaplan, G. (2000). Socioeconomic position: Social Epidemiology. New York: Oxford University Press.

Martin, J., \& Deacon, K. (2000). Development of a self-coded version of the new National Statistics Socio-economic classification (NS-SEC): preliminary results. SOCIAL SURVEY METHODOLOGY BULLETIN, 33-42.

Mohammad, K., Golestan, B., Majdzadeh, R., Chaman, R., Nedjat, S., \& Karimloo, M. (2009). Socioeconomic Status and Obesity Relationship in Non-Menopause Women Aged 15-49 Years in Tehran, Iran. Iranian Journal of Public Health, 38(3), 84-89.

Nagelhout, G. E., de Korte-de Boer, D., Kunst, A. E., van der Meer, R. M., de Vries, H., van Gelder, B. M., \& Willemsen, M. C. (2012). Trends in socioeconomic inequalities in smoking prevalence, consumption, initiation, and cessation between 2001 and 2008 in the Netherlands. Findings from a national population survey. BMC Public Health, 12(1), 1.

Nakhaee, N., Divsalar, K., Meimandi, M. S., \& Dabiri, S. (2008). Estimating the prevalence of opiates use by unlinked anonymous urine drug testing: a pilot study in Iran. Substance use \& misuse, 43(3-4), 513-520.

Nazarzadeh, M., Bidel, Z., Ayubi, E., Bahrami, A., Jafari, F., Mohammadpoorasl, A., . . Taremian, F. (2013). Smoking status in Iranian male adolescents: A cross-sectional study and a metaanalysis. Addictive behaviors, 38(6), 2214-2218.

Probst, C., Roerecke, M., Behrendt, S., \& Rehm, J. (2014). Socioeconomic differences in alcoholattributable mortality compared with all-cause mortality: a systematic review and metaanalysis. International journal of epidemiology, 43(4), 1314-1327. 
Riggs, N. R., \& Pentz, M. A. (2016). Inhibitory control and the onset of combustible cigarette, ecigarette, and hookah use in early adolescence: the moderating role of socioeconomic status. Child Neuropsychology, 22(6), 679-691.

Room, R., Sankaran, S., Schmidt, L., Mäkela, P., \& Rehm, J. (2015). Addictive substances and socioeconomic development The impact of addictive substances and behaviours on individual and societal well-being (pp. 189-214): Oxford University Press, Oxford.

Room, R., Sujatha Sankaran, L. A., Schmidt, P. M., \& Rehm, J. (2015). Addictive substances and socioeconomic development. The Impact of Addictive Substances and Behaviours on Individual and Societal Well-being, 189.

Ziaaddini, H., \& Ziaaddini, M. R. (2005). The household survey of drug abuse in Kerman, Iran. Journal of Applied Sciences, 5(2), 380-382.

Ziaaddini, H., Ziaaddini, T., \& Nakhaee, N. (2013). Pattern and trend of substance abuse in eastern rural Iran: A household survey in a rural community. Journal of addiction, 2013. 
